\title{
VISA, hetero-VISA and VRSA: The end of the vancomycin era?
}

\author{
John M Conly $M D^{1}$, B Lynn Johnston $M D^{2}$
}

$S^{n}$ taphylococcus aureus is a ubiquitous organism in the Shuman population $-30 \%$ to $40 \%$ of adults are asymptomatic carriers (1). As a species, staphylococci are among the most hardy of bacteria. They survive many adverse environmental conditions including heat, desiccation and relative cold, and they tolerate high salt concentrations (2). It is therefore not surprising that $S$ aureus has remained a major pathogen in humans, colonizing and infecting both hospitalized patients with reduced immune defences and healthy individuals with intact immune defences, but who have breaches to the normal skin and mucous membrane barriers. $S$ aureus also illustrates the dynamic interaction between pathogenic microorganisms and antimicrobials. The isolation of $S$ aureus strains that are resistant to penicillin $G$ was reported shortly after penicillin became widely available (3). Initially a sporadic occurrence, this type of resistance, which is plasmid borne, spread rapidly through the 1950 s and 1960s. By the 1970s, almost all hospital strains and a large number of community strains of $S$ aureus were resistant to penicillin. The development of the semisynthetic penicillinase-resistant agents, methicillin and the isoxazolyl penicillins in 1960 was followed by the report of methicillin-resistant S aureus (MRSA) in 1961 (4). There are several mechanisms of methicillin resistance in staphylococci, including inactivation by beta-lactamase enzymes, penicillin-binding proteins with reduced penicillin binding capacity, and the acquisition of the mecA gene, which encodes new penicillin-binding proteins with low affinity for beta-lactams. The latter mechanism accounts for the majority of resistance to methicillin and other beta-lactams (5).

The prevalence of MRSA in hospitalized patients was less than $5 \%$ in the early 1970s in most hospital settings worldwide, but within a decade, the prevalence had increased to as high as $40 \%$ in many hospitals in the United
States and Europe $(6,7)$. In the past 10 to 15 years, MRSA has become increasingly recognized as a major cause of hospital-acquired infections (8). Although MRSA is not more virulent than methicillin-sensitive Staphylococcus aureus, there is some evidence that infections such as pneumonia, endocarditis and cellulitis with MRSA create management difficulties due to delays in recognizing the presence of a resistant strain and cross-resistance to a number of commonly used antibiotics (5). These include most betalactams such as penicillins, cephalosporins and carbapenems, as well as many non-beta-lactam agents. The major risk factors for MRSA colonization include prolonged hospitalization, admission to an intensive care or burn unit, previous antimicrobial drug use and contact with a known MRSA carrier. Vancomycin is the treatment of choice for serious MRSA infections.

With the increasing prevalence of MRSA worldwide and the consequent increased use of vancomycin, it was inevitable that infections with vancomycin-resistant S aureus (VRSA) would be reported. The first clinical isolate of $S$ aureus with reduced susceptibility to vancomycin was reported from Japan in 1996 (9). The strain, known as $\mathrm{Mu} 50$, had a minimum inhibitory concentration (MIC) to vancomycin of $8 \mathrm{mg} / \mathrm{L}$, which is in the intermediate range based on interpretive criteria according to the National Committee for Clinical Laboratory Standards (NCCLS) (10). Following reports of the emergence of vancomycinintermediate $S$ aureus (VISA), another type of vancomycin resistance in S aureus was reported from Japan in 1997 (11) and was termed hetero-VISA. These strains are susceptible to vancomycin $(\mathrm{MIC}<4 \mathrm{mg} / \mathrm{L})$, but contain subpopulations of organisms that are capable of growing at a vancomycin concentration of $>4 \mathrm{mg} / \mathrm{L}$ and have an MIC of $\geq 8 \mathrm{mg} / \mathrm{L}$. In July 2002, the first documented report of clinical infection

\footnotetext{
${ }^{1}$ Departments of Pathology and Laboratory Medicine, Medicine, and Microbiology $\mathcal{E}$ Infectious Diseases, University of Calgary, Calgary, Alberta; ${ }^{2}$ Queen Elizabeth II Health Sciences Centre and Dalhousie University, Halifax, Nova Scotia

Correspondence: Dr John Conly, Departments of Medicine, Pathology and Laboratory Medicine, and Microbiology 83 Infectious Diseases, Department of Medicine, Room 930, 9th Floor, North Tower, 1403-29th Street Northwest, Calgary, Alberta T2N 2T9. Telephone 403-944-8222, fax 403-944-1095, e-mail jconly@ucalgary.ca and Dr Lynn Johnston, Room 5014 ACC, Queen Elizabeth II Health Sciences Centre, 1278 Tower Road, Halifax, Nova Scotia B3H 2Y9. Telephone 902-473-8477, fax 902-473-7394, e-mail ljohnsto@is.dal.ca
} 
caused by $S$ aureus that was fully resistant to vancomycin was published in the United States (12).

The nomenclature and clinical significance of vancomycin resistance in $S$ aureus can be confusing. Some of this confusion arises over different breakpoints used in the literature, which can vary according to the reporting country and with the use of differing terms to describe the phenomenon of vancomycin heteroresistance in subpopulations of $S$ aureus. In addition, commonly used laboratory techniques for determining antibiotic susceptibility to vancomycin have proved to be less than optimal for detecting VISA and hetero-VISA. Most laboratories in Canada follow the guidelines set out by the NCCLS (10), in which staphylococci with an MIC of $\leq 4 \mathrm{mg} / \mathrm{L}$ to vancomycin are considered to be susceptible, those with an MIC of 8 to 16 $\mathrm{mg} / \mathrm{L}$ are intermediate and those strains with an MIC of $\geq 32$ $\mathrm{mg} / \mathrm{L}$ are resistant. Disk diffusion testing has been found to misclassify VISA as fully susceptible (13), and automated testing methods such as MicroScan (Dade Behring Inc, USA) and Vitek (BioMérieux Inc, USA) have failed to identify VISA strains $(13,14)$. At present, MIC determinations using broth dilution, agar dilution or E-test are considered to be the best methods for detecting reduced susceptibility to vancomycin $(14,15)$. The Centers for Disease Control and Prevention (16,17) have published recommendations to guide laboratories in the susceptibility testing of S aureus isolates to detect increased MICs to vancomycin. Although a VISA strain would be expected to exhibit an MIC of $\geq 8 \mathrm{mg} / \mathrm{L}$, the hetero-VISA strain has generally required formal population analysis using the serial passage of screened isolates of $S$ aureus on selective agar containing increasing concentrations of vancomycin for detection (18). This type of testing is beyond the scope of most clinical laboratories.

VISA clinical isolates have been reported from Japan, the United States (Michigan, New Jersey, New York and Illinois), the United Kingdom, France and Hong Kong $(9,19-25)$, and clinical failures to vancomycin treatment have been well documented with these isolates. HeteroVISA strains have been reported from Japan, Hong Kong, Spain, Italy, Germany and Korea (11,25-29). The identification of hetero-VISA has suggested that some $S$ aureus strains regarded as 'susceptible' by MIC testing may represent a continuum between VISA isolates and truly susceptible populations of $S$ aureus. The prevalence of hetero-VISA varies and appears to be dependent on the population and geographical area. In the study from Spain (26), in which only MRSA isolates from surgical site infections in orthopedic patients were screened, $65 \%$ of the strains had subpopulations with elevated MICs to vancomycin. At present, the clinical significance of hetero-VISA is not understood fully. One retrospective study suggested that patients who were infected with hetero-resistant strains causing bacteremia had a higher mortality rate than patients who were infected with fully sensitive strains (30), and another study suggested a higher failure rate with vancomycin in patients with surgical site infections (26). However, other studies have not found any relationship between hetero-VISA and treatment failure with vancomycin (29).

The relationship between hetero-VISA and VISA is interesting, however, and several lines of evidence suggest that hetero-VISA strains give rise to VISA after prolonged exposure to glycopeptides. Clonal relationships have been reported between hetero-VISA and VISA strains isolated from the same hospital or region $(31,32)$, and between a vancomycin-susceptible MRSA and a VISA strain isolated from the same patient $(33,34)$. In vitro, the level of vancomycin resistance of hetero-VISA has been reported to be increased by exposure to higher concentrations of vancomycin in a stepwise fashion (34).

The mechanism of resistance in hetero-VISA and VISA involves a complex reorganization of cell wall metabolism, leading to a grossly thickened cell wall with reduced glycopeptide cross-linking $(33,34)$. Before the identification of this finding in clinical isolates of VISA, laboratory-induced strains of VISA were noted to have thicker cell walls than the parent strains (35). It has been proposed that the thickened cell walls may trap and sequester vancomycin and, thus, limit its effect by preventing penetration to its site of action $(36,37)$.

The mechanism of resistance described for the first clinical isolate of VRSA reported in the United States (12) was related to the presence of the vanA vancomycin resistance gene, which has been described in enterococci. The MIC for vancomycin for this strain was $>128 \mathrm{mg} / \mathrm{L}$ by the broth microdilution method. The presence of the vanA gene in this isolate, the presence of a vancomycin-resistant Enterococcus fecalis in the wound of the patient and the previously demonstrated in vitro conjugative transfer of the vanA gene from enterococci to $S$ aureus (38) suggest that this latter phenomenon occurred in the clinical setting.

While no isolates of $S$ aureus with intermediate or complete resistance to vancomycin have been described in Canada, there is a need for continued vigilance within clinical microbiology laboratories. The appearance of heteroVISA and VISA appears to be more common given the number of reports in the literature. It is interesting to note that, despite the coexistence of MRSA and vancomycinresistant enterococci in the clinical setting for more than 14 years in the United States, VRSA has not appeared until now. Although the event may be rare, once a patient becomes colonized, the major concerns are the horizontal transfer between patients and the establishment of an endemic focus. Unfortunately, little is known about the epidemiology or risks for the horizontal transfer of heteroVISA and VISA. This is only the beginning of the era of vancomycin resistance in $S$ aureus and other staphylococcal species. Much work must be done to fully elucidate the mechanisms of resistance, the risk factors for their occurrence, the optimal management of patients who are infected with these organisms and, ultimately, how best to prevent them from spreading. 


\section{REFERENCES}

1. Lowey FD. Staphylococcus aureus infections. N Engl J Med 1998;339:520-32.

2. Clements MO, Foster SJ. Stress resistance in Staphylococcus aureus. Trends Microbiol 1997:11:458-62.

3. Spink WW, Ferris V. Quantitative action of penicillin inhibitor from penicillin-resistant strains of staphylococci. Science 1945;102:221.

4. Barber M. Methicillin-resistant staphylococci. J Clin Pathol 1961:14:385.

5. Brumfitt W, Hamilton-Miller JMT. Methicillin-resistant Staphylococcus aureus. N Engl J Med 1989;320:1188-96.

6. Panlilio AL, Culver DG, Gaynes RP, et al. Methicillin-resistant Staphylococcus aureus in US hospitals, 1975-1991. Infect Control Hosp Epidemiol 1992;13:582-6.

7. Voss A, Milatovic D, Wallrauch-Schwarz C, et al. Methicillinresistant Staphylococcus aureus in Europe. Eur J Clin Microbiol Infect Dis 1994;13:50-5.

8. Centers for Disease Control and Prevention. National nosocomial infections surveillance report - data summary from October 1986April 1996, issued May 1996. Am J Infect Control 1996;24:380-8.

9. Hiramatsu K, Hanaki H, Ino T, Yabuta K, Oguri T, Tenover FC. Methicillin-resistant Staphylococcus aureus clinical strain with reduced vancomycin susceptibility. J Antimicrob Chemother 1997:40:135-6

10. National Committee for Clinical Laboratory Standards. Methods for dilution antimicrobial susceptibility tests for bacteria that grow aerobically. NCCLS approved standard M7-A5. Wayne: National Committee for Laboratory Standards, 2000.

11. Hiramatsu K, Aritaka N, Hanaki H, et al. Dissemination in Japanese hospitals of strains of Staphylococcus aureus heterogeneously resistant to vancomycin. Lancet 1997;350:1670-3.

12. Centers for Disease Control and Prevention. Staphylococcus aureus resistant to vancomycin - United States, 2002. MMWR Morb Mortal Wkly Rep 2002;51:565-7.

13. Tenover FC, Lancaster MV, Hill BC, et al. Characterization of staphylococci with reduced susceptibilities to vancomycin and other glycopeptides. J Clin Microbiol 1998;36:1020-7.

14. Tenover FC, Mohammed MJ, Stelling J, O'Brien T, Williams R. Ability of laboratories to detect emerging antimicrobial resistance: Proficiency testing and quality control results from the World Health Organization's external quality assurance system for antimicrobial susceptibility testing. J Clin Microbiol 2001;39:241-50.

15. Tenover FC, Biddle JW, Lancaster MV. Increasing resistance to vancomycin and other glycopeptides in Staphylococcus aureus. Emerg Infect Dis 2001;7:327-32

16. Centers for Disease Control and Prevention. Interim guidelines for prevention and control of staphylococcal infection associated with reduced susceptibility to vancomycin. MMWR Morb Mortal Wkly Rep 1997;46:626-8.

17. Centers for Disease control and Prevention. Staphylococcus aureus with reduced susceptibility to vancomycin - Illinois. MMWR Morb Mortal Wkly Rep 1999;48:1165-7.

18. Boyle-Vavra S, Carey RB, Daum RS. Development of lysostapin and vancomycin resistance in pre-GISA vancomycin-tolerant, methicillin-resistant Staphylococcus aureus isolate. J Antimicrob Chemother 2001;48:617-25.

19. Centers for Disease Control and Prevention. Staphylococcus aureus with reduced susceptibility to vancomycin - United States, 1997. MMWR Morb Mortal Wkly Rep 1997;46:765-6.
20. Rotun SS, McMath V, Schoonmaker DJ, et al. Staphylococcus aureus with reduced susceptibility to vancomycin isolated from a patient with fatal bacteremia. Emerg Infect Dis 1999;5:147-9.

21. Sieradzki K, Roberts R, Haber S, Tomasz A. The development of vancomycin resistance in a patient with methicillin-resistant Staphylococcus aureus. N Engl J Med 1999;340:517-23.

22. Centers for Disease Control and Prevention. Staphylococcus aureus with reduced susceptibility to vancomycin - Illinois, 1999. JAMA 2000;283:597-8.

23. Howe RA, Bowker KE, Walsh TR, Feest TG, MacGowan AP. Vancomycin-resistant Staphylococcus aureus. Lancet 1998;351:602.

24. Ploy MC, Grelaud C, Martin C, deLumley L, Denis F. First clinical isolate of vancomycin-intermediate Staphylococcus aureus in a French hospital. Lancet 1998;351:1212.

25. Wong SS, Ng TK, Yam WC. Bacteremia due to Staphylococcus aureus with reduced susceptibility to vancomycin. Diagn Microbiol Infect Dis 2000;36:261-8.

26. Ariza J, Pujol M, Cabo J, et al. Vancomycin in surgical infections due to methicillin-resistant Staphylococcus aureus with heterogeneous resistance to vancomycin. Lancet 1999;353:1587-8.

27. Marchese A, Balistreri G, Tonoli E, Bebbia EA, Schito GC. Heterogeneous vancomycin-resistance in methicillin-resistant Staphylococcus aureus strains in a large Italian hospital. J Clin Microbiol 2000;38:866-9.

28. Geisel R, Schmitz FJ, Thomas L, et al. Emergence of heterogeneous intermediate vancomycin-resistance in Staphylococcus aureus isolates in the Dusseldorf area. J Antimicrob Chemother 1999;43:846-8.

29. Kim M-N, Hwang SH, Pyo Y-J, et al. Clonal spread of Staphylococcus aureus heterogenously resistant to vancomycin in a university hospital in Korea. J Clin Microbiol 2002;30:1376-80.

30. Wong SS, Ho PL, Woo PC, Yuen KY. Bacteremia caused by staphylococci with inducible vancomycin heteroresistance. Clin Infect Dis 1999;29:760-7.

31. Hiramatsu K, Aritaka N, Hanaki H, et al. Dissemination in Japanese hospitals of strains of Staphylococcus aureus heterogeneously resistant to vancomycin. Lancet 1997;350:1670-3.

32. Bierbaum Gl, Fuchs K, Lenz W, Szekat C, Gsahl H. Presence of Staphylococcus aureus with reduced susceptibility to vancomycin in Germany. Eur J Microbiol Infect Dis 1999;18:691-6.

33. Sieradzki K, Roberts RB, Haber SW, Tomasz A. The development of vancomycin resistance in a patient with methicillin-resistant Staphylococcus aureus infection. N Engl J Med 1999;340:519-23.

34. Smith TL, Pearson ML, Wilcox KR, et al. Emergence of vancomycin resistance in Staphyococcus aureus. N Engl J Med 1999;340:493-501.

35. Howe RA, Wooton M, Walsh TR, Bennet PM, MacGowan AP. Expression and detection of hetero-vancomycin resistance in Staphylococcus aureus. J Antimicrob Chemother 1999;44:675-8.

36. Daum RS, Gupta S, Sabbagh R, Milewski WM. Characterization of Staphylococcus aureus isolates with decreased susceptibility to vancomycin and teicoplanin: Isolation and purification of a constitutively produced protein associated with decreased susceptibility. J Infect Dis 1992;166:1066-72.

37. Sieradzki K, Tomasz A. Inhibition of cell wall turnover and autolysis by vancomycin in a highly vancomycin-resistant mutant of Staphylococcus aureus. J Bacteriol 1997;179:2557-66.

38. Noble WC, Virani Z, Cree RG. Co-transfer of vancomycin and other resistance genes from Enteroccus faecalis NCTC 12201 to Staphylococcus aureus. FEMS Microbiol Lett 1992;93:195-8. 


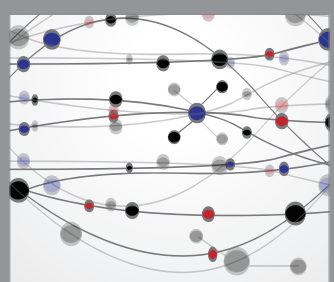

The Scientific World Journal
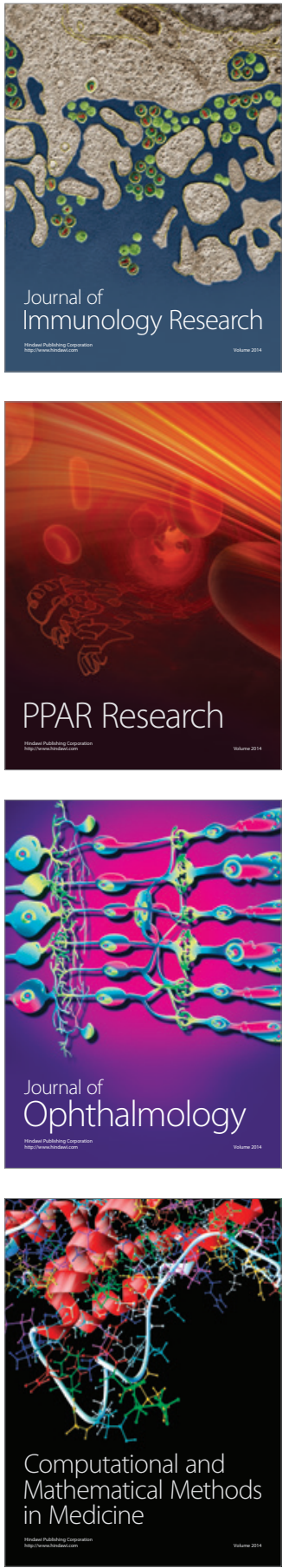

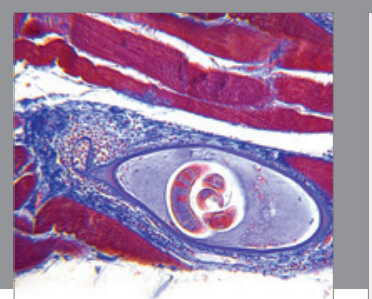

Gastroenterology Research and Practice

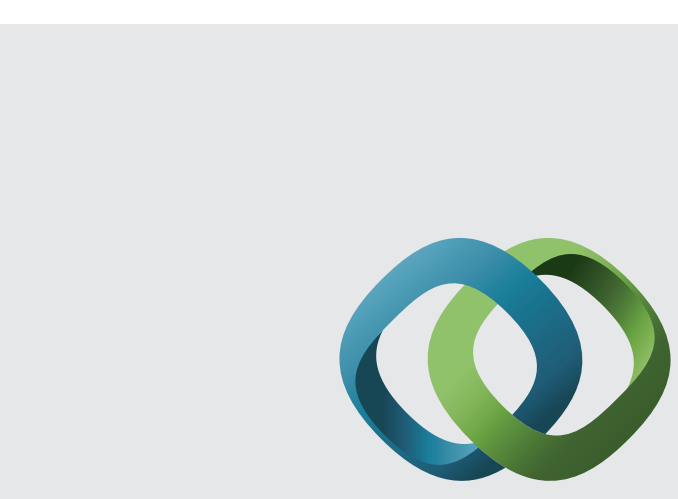

\section{Hindawi}

Submit your manuscripts at

http://www.hindawi.com
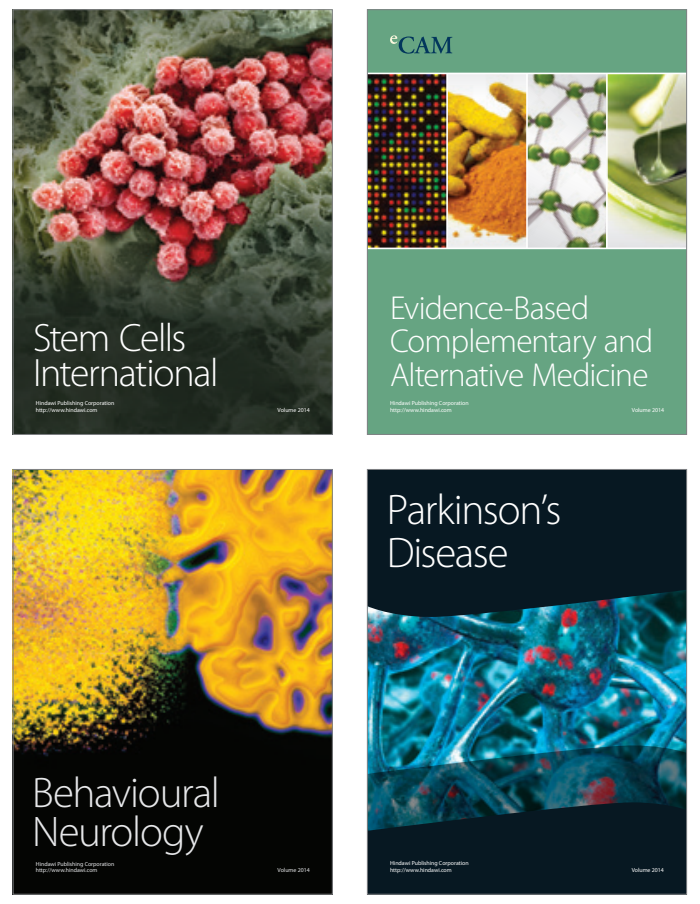
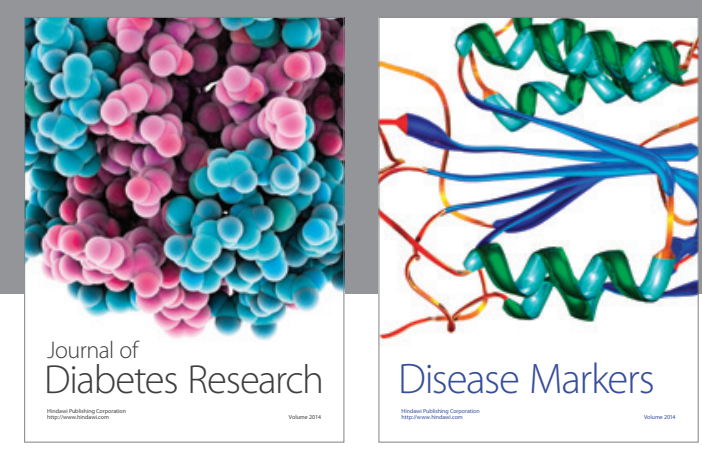

Disease Markers
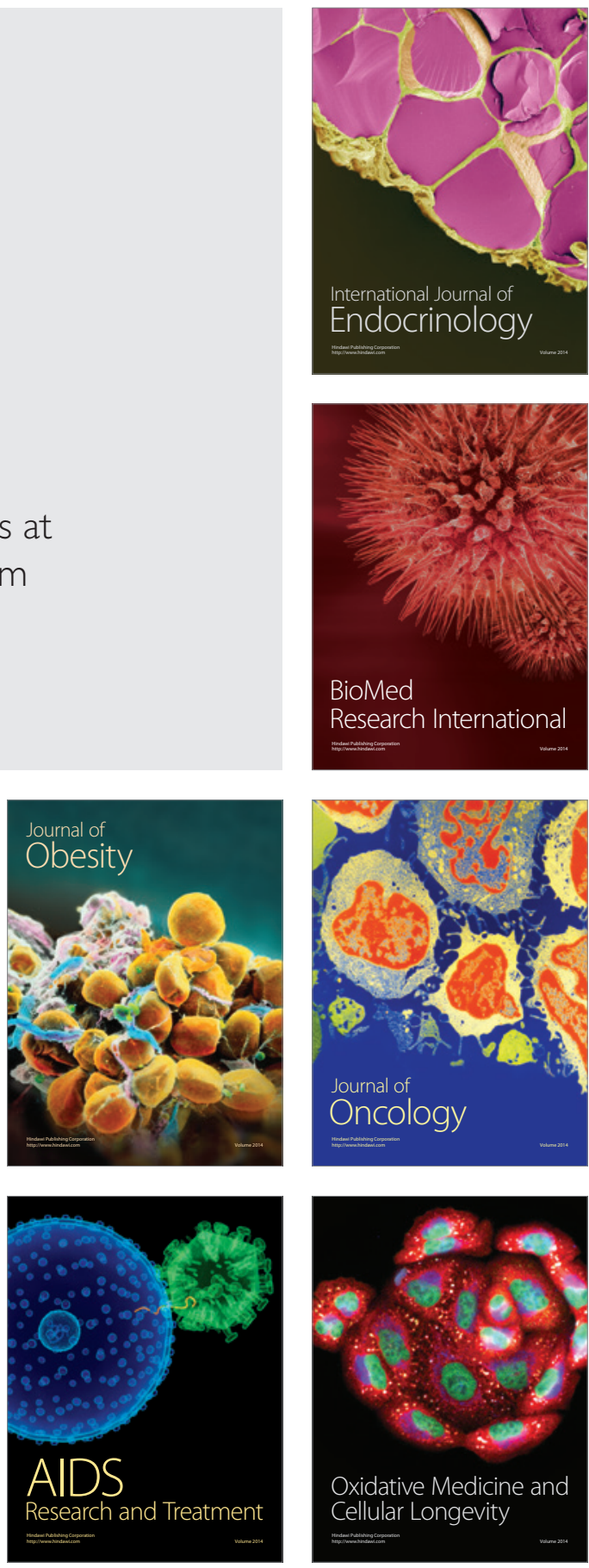